\title{
Contralateral Acute Subdural Hematoma Developing Intraoperatively following Evacuation of an Ipsilateral Spontaneous Acute Subdural Hematoma in an Elderly Patient
}

\author{
Rajesh Bhosle ${ }^{1}$ Dimble Raju $\quad$ Shamshuddin Pate \\ ${ }^{1}$ Department of Neurosurgery, National Neurosciences Centre, \\ Kolkata, West Bengal, India \\ 2Department of Neuroanesthesiology, National Neurosciences \\ Centre, Kolkata, West Bengal, India
}

Indian J Neurotrauma 2021;18:150-151.

A 73-year-old male patient presented in an unconscious state with weakness of the right side of the body, 8 hours after a fall. On examination, he had no eye opening, no verbal response, and on pain was localizing with the left hand. He was flexing weakly with the right upper limb on pain. There was no history of antiplatelet or anticoagulant intake and he had no other preexisting medical illnesses. Computed tomography (CT) scan showed a left-sided acute subdural hematoma (SDH) with significant mass effect, effacement of cisterns, and midline shift ( $\mathbf{F}$ Fig. 1A-C). There was no contralateral intracranial bleed or injury. He underwent left-sided temporoparietal craniotomy, durotomy, and clot evacuation. Intraoperatively, an arterial bleeding point was noted in the left frontal lobe and was cauterized. The brain was extremely lax and hence after duraplasty, the bone flap was replaced. Postoperatively, he was reversed from anesthesia but not extubated and was noted to have left-sided focal seizures. A CT scan was done that showed contralateral acute SDH with a small interhemispheric SDH with no parenchymal contusion ( - Fig. 2A-E). However, as this was not causing any significant midline shift or cisternal effacement conservative treatment was continued. A magnetic resonance imaging (MRI) study was done later too that showed only an acute subdural hematoma on the right side with no cerebral injury ( - Fig. 3A-D). His seizures were managed with anticonvulsants and serial CT scans showed resolution of the contralateral SDH over time ( - Fig. 3E-H). He was discharged uneventfully after 3 weeks with a full recovery.

\section{Discussion}

Contralateral hematomas after cranial surgeries are rare but potentially life-threatening complications. ${ }^{1-3}$ The most

published online

July 29, 2021
DoI https://doi.org/

$10.1055 / \mathrm{s}-0041-1732788$ ISSN 0973-0508

\author{
Nabanita Ghosh ${ }^{2}$ Prasad Krishnan ${ }^{1, \odot}$
}

Address for correspondence Prasad Krishnan, MS, MCh, Department of Neurosurgery, National Neurosciences Centre, Second Floor, Peerless Hospital Campus, 360, Panchasayar, Garia, Kolkata 700094, West Bengal, India (e-mail: prasad.krishnan@rediffmail.com).

commonly described type of contralateral hematoma is an extradural hemorrhage. ${ }^{4}$ Contralateral intracerebral hemorrhages (usually expansion of a preexisting contusion) are the second commonest ${ }^{5}$ and contralateral acute SDH is the rarest. Most of the reports in literature on development of a contralateral acute SDH after operation are in the nature of case reports. However Chrastina et $\mathrm{al}^{6}$ found a contralateral acute SDH, necessitating reoperation in 5 out of 86 cases operated for an acute SDH while Shibahashi et $\mathrm{al}^{7}$ have stated that the incidence and mortality of contralateral acute SDH after ipsilateral acute SDH evacuation to be 4.1 and $75 \%$, respectively.

Sudden shift of the contralateral hemisphere toward the side of the craniotomy after decompression with rupture of bridging veins, ${ }^{2}$ bleeding from a previously undetected contusion, ${ }^{8}$ release of tamponade of a contralateral bleeding point, ${ }^{2,9}$ sudden increase in blood flow in the contralateral side after rapid release of intracranial pressure at the time of decompression, ${ }^{2,8,10}$ bleeding diathesis secondary to coagulopathy or due to intake of antiplatelet or anticoagulant medications, ${ }^{2}$ faulty positioning during surgery causing cerebral

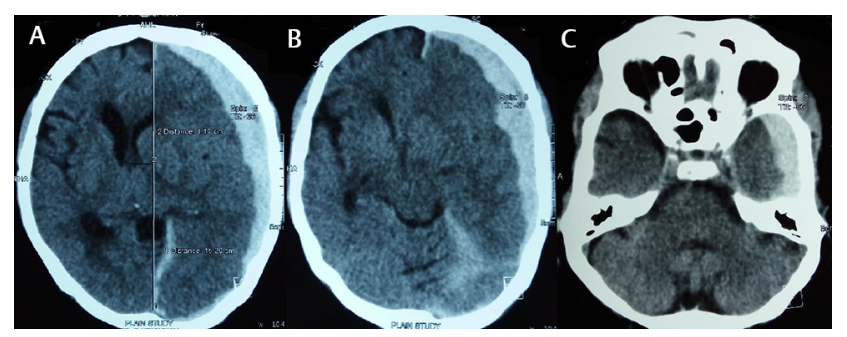

Fig. 1 Preoperative computed tomography (CT) scan images (A-C) showing left-sided acute subdural hematoma with significant mass effect and midline shift with no contralateral contusion or acute subdural hematoma seen.

\section{(C) 2021. Neurotrauma Society of India.}

This is an open access article published by Thieme under the terms of the Creative Commons Attribution-NonDerivative-NonCommercial-License, permitting copying and reproduction so long as the original work is given appropriate credit. Contents may not be used for commercial purposes, or adapted, remixed, transformed or built upon. (https://creativecommons.org/licenses/by-nc-nd/4.0/).

Thieme Medical and Scientific Publishers Pvt. Ltd. A-12, 2nd Floor, Sector 2, Noida-201301 UP, India 


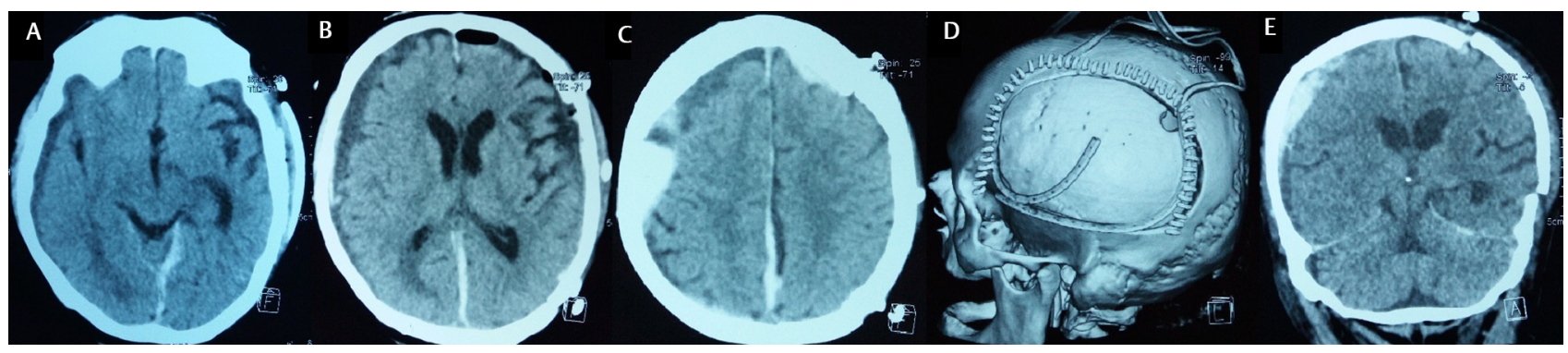

Fig. 2 Postoperative computed tomography (CT) scan images (A-C) showing complete evacuation of the subdural hematoma on the left side and a fresh subdural hematoma on the right side with no obvious contusion; (D) reconstructed three-dimensional image showing replaced bone flap on the left side and (E) coronal CT image showing a convexity acute subdural hematoma on the right side.

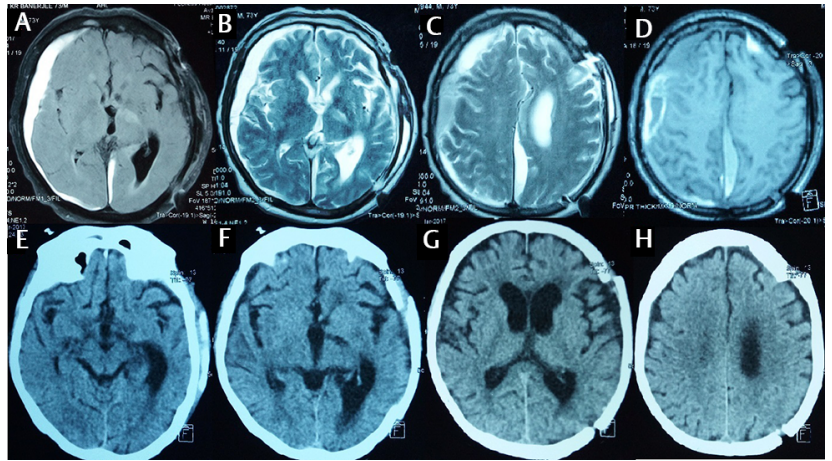

Fig. 3 Postoperative MRI (A) FLAIR images showing a hyperintense collection over the right cerebral convexity and an interhemispheric collection; (B, C) T2-weighted images and (D) T1-weighted images showing blood over the right cerebral convexity with no evidence of any parenchymal injury; postoperative CT scan images after 4 weeks (E-H) showing complete resolution of the right-sided acute subdural hematoma. CT, computed tomography; MRI, magnetic resonance imaging.

venous hypertension (due to excessive neck rotation), ${ }^{2,8}$ and overuse of osmotic dehydrating agents ${ }^{9}$ have all been postulated as reasons for development of a fresh contralateral acute SDH.

Most cases described in literature underwent decompressive craniotomy initially ${ }^{1,29}$ for the acute SDH where the potential of contralateral hemispheric shift toward the craniectomy defect is greater, unlike in our case where the bone flap had been replaced. Suspicion of a contralateral pathology was precipitated in our case due to development on focal seizures on the same side as the craniotomy while other cases in literature were unmasked following imaging necessitated by unexplained neurological worsening or inexplicable intraoperative brain bulge. . $^{1-3,5,9}$

\section{Conclusion}

It must be remembered that whatever the pathogenesis, contralateral acute SDH is more likely in elderly patients than an extradural hematoma since their dura is intimately adherent to the skull, and further these patients have brain atrophy with a potentially larger subdural space where the bridging veins may rupture as the brain shifts intraoperatively. It is mandatory to have a low threshold for early postoperative imaging in elderly patients, those with unexpected intraoperative brain bulge or inexplicable postoperative deficits and in those, where there is suspicion of contralateral associated intracranial injuries for early detection and treatment of this complication.

\section{Conflict of Interest \\ None declared.}

\section{References}

1 Takeuchi S, Takasato Y. Contralateral acute subdural hematoma after surgical evacuation of the initial hematoma: two case reports and review of the literature. Turk Neurosurg 2013;23(2):294-297

2 Shen J, Fan Z, Ji T, Pan J, Zhou Y, Zhan R. Contralateral acute subdural hematoma following traumatic acute subdural hematoma evacuation. Neurol Med Chir (Tokyo 2013;53(4):221-224

3 Lv J, Qi X, Wang Y, et al. Contralateral subdural hematoma following surgical evacuation of acute subdural hematoma: super-early intervention and clinical implications. World Neurosurg 2019;122:24-27

4 Fridley J, Thomas J, Kitagawa R, Chern J, Omeis I. Immediate development of a contralateral acute subdural hematoma following acute subdural hematoma evacuation. J Clin Neurosci 2011;18(3):422-423

5 Choi YH, Lim TK, Lee SG. Clinical features and outcomes of bilateral decompression surgery for immediate contralateral hematoma after craniectomy following acute subdural hematoma. Korean J Neurotrauma 2017;13(2):108-112

6 Chrastina J, Silar C, Zeman T, et al. Reoperations after surgery for acute subdural hematoma: reasons, risk factors, and effects. Eur J Trauma Emerg Surg 2020;46(2):347-355

7 Shibahashi K, Hoda H, Takasu Y. Contralateral subdural hematoma development following unilateral acute subdural hematoma evacuation. Br J Neurosurg 2017;31(5):619-623

8 Pradhan RR, Shrestha GS, Sedain G. Remote supratentorial subdural hematoma following craniectomy and evacuation of hypertensive cerebellar hematoma. Cureus 2020;12(2):e6977

9 Satyarthee GD, Gaurang V, Sharma BS. Contralateral development of massive acute subdural hematoma occurrence during decompressive craniectomy and surgery for evacuation of ipsilateral acute subdural hematoma: literature review. IJNT 2014;11:118-121

10 Tomycz ND, Germanwala AV, Walter KA. Contralateral acute subdural hematoma after surgical evacuation of acute subdural hematoma. J Trauma 2010;68(1):E11-E12 